\title{
X-ray structure of the dopamine transporter in complex with tricyclic antidepressant
}

\author{
Aravind Penmatsa ${ }^{1,{ }^{*}, \text { Kevin H. Wang }}{ }^{1,{ }^{*}, \text { and Eric Gouaux }}{ }^{1,2, \#}$ \\ ${ }^{1}$ Vollum Institute, Oregon Health \& Science University, 3181 SW Sam Jackson Park Road, \\ Portland, OR 97239 \\ ${ }^{2}$ Howard Hughes Medical Institute, Oregon Health \& Science University, 3181 SW Sam Jackson \\ Park Road, Portland, OR 97239
}

\section{Abstract}

Antidepressants targeting $\mathrm{Na}^{+} / \mathrm{Cl}^{-}$-coupled neurotransmitter uptake define a major therapeutic strategy to treat clinical depression and neuropathic pain. However, identifying the molecular interactions that underlie the pharmacological activity of these transport inhibitors and thus the mechanism by which the inhibitors lead to increased synaptic neurotransmitter levels has proven elusive. Here we present the crystal structure of the Drosophila melanogaster dopamine transporter (dDAT) at $3.0 \AA$ A resolution bound to the tricyclic antidepressant nortriptyline. The transporter is locked in an outward-open conformation with nortriptyline wedged between TMs1/6 and $3 / 8$, blocking the transporter from binding substrate and from isomerizing to an inward facing conformation. While the overall structure of dDAT is similar to that of its prokaryotic relative LeuT, there are multiple distinctions that include a kink in TM12 halfway across the membrane bilayer, a latch-like $\mathrm{C}$-terminal helix that caps the cytoplasmic gate, and a cholesterol molecule wedged within a groove formed by TMs 1a, 5 and 7 . Taken together, the dDAT structure reveals the molecular basis for antidepressant action on sodium-coupled neurotransmitter symporters and illuminates critical elements of eukaryotic transporter structure and modulation by lipids, thus expanding our understanding of mechanism and regulation of neurotransmitter uptake at chemical synapses.

\section{Introduction}

Chemical neurotransmission involves release of neurotransmitters upon $\mathrm{Ca}^{2+}$-induced depolarization of presynaptic nerve cells ${ }^{1}$. Upon release into the synaptic cleft, neurotransmitters such as glutamate, dopamine, norepinephrine, serotonin, glycine, and GABA ( $\gamma$-aminobutyric acid) activate GPCRs and ligand-gated ion channels resulting in excitatory or inhibitory postsynaptic signaling cascades and currents ${ }^{1-3}$. The widespread and critical roles played by neurotransmitters in both central and peripheral nervous systems necessitate a requirement for strict spatio-temporal control of their levels at neural synapses. The primary mode of neurotransmitter clearance from the synaptic cleft is through

\footnotetext{
\#CORRESPONDING AUTHOR Correspondence and requests for materials should be addressed to E.G. (gouauxe@ ohsu.edu). TEL: (503) 494-5535, FAX: (503) 494-1700.

These authors contributed equally to this work.

Author Contributions

A.P., K.W., and E.G. designed the project. A.P. and K.H.W. performed protein purification, crystallography, and biochemical assays. A.P., K.H.W., and E.G. wrote the manuscript.

The coordinates for the structure have been deposited in the Protein Data Bank under the accession code xxxx. Reprints and permissions information is available at www.nature.com/reprints. The authors declare no competing financial interests.
} 
secondary active transporters localized in presynaptic and glial cells that harness ionic gradients, across the cell membrane, to drive the uphill transport of neurotransmitters ${ }^{4}$. This symport process requires both $\mathrm{Na}^{+}$and $\mathrm{Cl}^{-}$ions ${ }^{5}$, which therefore, has led to the solute carrier 6 (SLC6) family of secondary transporters ${ }^{3}$, to be referred to as neurotransmitter sodium symporters (NSSs) ${ }^{2}$.

Dysregulation of NSS function is associated with several debilitating disorders that include depression $^{2}$, attention deficit hyperactivity disorder (ADHD) ${ }^{6}$, orthostatic intolerance ${ }^{7}$, epilepsy $^{8}$, Parkinson's disease ${ }^{2}$ and infantile parkinsonism dystonia ${ }^{9}$. NSSs are also the primary targets of antidepressants, drugs to treat neuropathic pain, ADHD, anxiety and habit-forming substances of abuse such as cocaine and amphetamines ${ }^{3}$. Development of antidepressants had a serendipitous beginning in the $1950 \mathrm{~s}^{10}$, followed by the discovery that the tricyclic antidepressant (TCA) imipramine, inhibits norepinephrine reuptake in tissues ${ }^{11}$. Numerous variants of imipramine, and the subsequent discovery of selective serotonin reuptake inhibitors have revolutionized antidepressant treatment ${ }^{12},{ }^{13}$. To date, inhibition of neurotransmitter uptake remains the most widely used strategy for antidepressant therapy ${ }^{12}$, despite numerous side-effects ${ }^{14}$.

Gains in our understanding of the molecular mechanisms underlying sodium-coupled transport have benefited from the structures of multiple conformations of LeuT ${ }^{15},{ }^{16},{ }^{17}$, a bacterial sodium-coupled amino acid transporter with $\sim 20 \%$ sequence identity to the eukaryotic NSSs. Models of eukaryotic NSSs based on LeuT have provided valuable insights into substrate and ion specificities, pharmacology, and transport mechanisms in NSS members ${ }^{18,19}$. However, bacterial NSS models fall short of answering questions concerning the elements of NSS structure and function that include the local structure of NSSs in regions that are unrelated to LeuT in amino acid sequence, the determinants of substrate selectivity and the atomic level details of transport inhibition by antidepressants and addictive compounds. Moreover, there is no understanding, at the level of threedimensional structure, on the role of lipids and post-translational modifications in NSS structure and mechanism.

Here we present a $3.0 \AA$ x-ray crystal structure of the Drosophila melanogaster dopamine transporter $(\mathrm{dDAT})^{20}$ in complex with the TCA nortriptyline. The dDAT has greater than $50 \%$ sequence identity with its mammalian counterparts and harbors a pharmacological profile that is a hybrid of the mammalian dopamine (DAT), norepinephrine (NET) and serotonin transporters (SERT), thus rendering dDAT a powerful vehicle to study NSS pharmacology and substrate specificity ${ }^{20}$. The dDAT structure reveals atomic details of TCA recognition, novel structural elements of NSS protein architecture and suggests a role for cholesterol in the allosteric control of transport in eukaryotic NSS members.

\section{Thermostabilization and crystallization}

Wild-type dDAT is labile, loses ligand binding activity upon detergent extraction from the cellular membranes and is refractory to crystallization. To stabilize dDAT for functional characterization, antibody generation and crystallization, we screened single point mutants for ligand binding activity at elevated temperatures ${ }^{21}$, ultimately combining 5 mutations into the construct used for crystallization and structure determination $\left(\mathrm{dDAT}_{\text {cryst }} ;\right.$ Supplementary Fig. 1). Purified dDAT cryst binds to the high-affinity inhibitor nisoxetine with a $K_{D}$ of 29 nM (Supplementary Fig. 2a), and the TCA nortriptyline exhibits a $K_{i}$ of $156 \mathrm{nM}$ (Supplementary Fig. 2b). Unfortunately we were unable to measure the binding of nortriptyline to wild-type dDAT because of its instability. Nortriptyline has a $K_{i}$ of $18 \mathrm{nM}$ at hSERT and $4.4 \mathrm{nM}$ at hNET ${ }^{22}$, values that are $\sim 9$-fold and $\sim 35$-fold lower than that for $\mathrm{dDAT}_{\text {cryst }}$. In dopamine uptake measurements with wild-type dDAT amitriptyline, a 
precursor of nortriptyline, inhibits wild-type dDAT transport with a $K_{i}$ of $30 \mathrm{nM}^{20}$, whereas the $\mathrm{dDAT}_{\text {cryst }}$ construct is inactive in transport (Supplementary Fig. 2c, d). Crystallization was further enhanced by the use of a complex with a Fab, resulting in crystals of a $\mathrm{dDAT}_{\text {cryst }}-\mathrm{Fab}$ complex that diffract X-rays to $3.0 \AA$ A resolution.

\section{Architecture of dDAT}

The structure of $\mathrm{dDAT}_{\text {cryst }}$ bound to nortriptyline exhibits an outward-open conformation whereby the antidepressant is bound in a cavity halfway across the membrane bilayer and accessible to solvent from only the extracellular side of the membrane (Fig. 1). The transporter displays an overall LeuT-like fold with 12 transmembrane (TM) helices where helices $1-5$ and 6-10 are related by inherent pseudosymmetry, akin to LeuT ${ }^{15}$

(Supplementary Fig. 3). Residues in TM1 and TM6 make numerous interactions with the ligand and ions via non helical, hinge-like regions at the approximate mid-points of these TMs, connecting the bonding networks of all three ions with the inhibitor. Residues at the bend in TM 3 contribute to the hydrophobic pocket that cradles the tricyclic moiety of the ligand, which lays approximately perpendicular to the TMs, mimicking a wedge separating the jaws of a vise. One cholesterol molecule is located in a groove between TM5 and TM7 and poised to modulate the movement of TM1a that occurs during the transport cycle (Fig. 1a) ${ }^{17}$.

The primary binding site accommodates nortriptyline but cannot adopt the subsequent helical movements of TMs $1 \mathrm{~b}$ and $6 \mathrm{a}$ required to form the occluded state. Using LeuT for comparison, the occluded state of LeuT is formed in the presence of sodium and leucine substrate, but not in the presence of tryptophan, which binds to the primary site, comparable

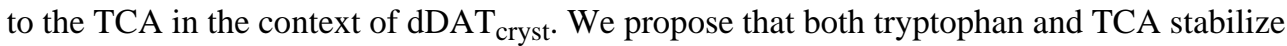
the outward-open conformations of LeuT and $\mathrm{dDAT}_{\text {cryst }}$, respectively, by targeting the primary binding site and sterically blocking the extracellular domains of the transporter, preventing the extracellular gate from closing and thus acting by way of a foot-in-the-door mechanism (Supplementary Fig. 4a, b; Supplementary Table 2).

Whereas the core of $\mathrm{dDAT}_{\text {cryst }}$ closely resembles that of LeuT, the periphery of $\mathrm{dDAT}_{\text {cryst }}$ exhibits several features distinct from LeuT and important for neurotransmitter transport and cellular localization. In TM12, a kink in the center at Pro 572 causes the second half of the helix to turn away from the transporter, indicating that the dimerization interface of LeuT is not the same as potential oligomerization interfaces of eukaryotic NSSs (Fig. 1a and b, Supplementary Fig. 5). While previous studies indicate that NSSs oligomerize ${ }^{23,24}$, $\mathrm{dDAT}_{\text {cryst }}$ is monomeric in detergent micelles and in the crystal lattice (Supplementary Fig. 6), thus suggesting that a membrane bilayer or additional molecules may be required for NSS assembly. The variable EL2 region has numerous predicted $N$-linked glycosylation sites $^{25}$ and one disulfide bond ${ }^{26}$, modifications that play critical roles in proper trafficking of NSSs to the plasma membrane ${ }^{3}$. The strictly conserved disulfide linkage ${ }^{26}$ was observed in the structure between two conserved cysteines, Cys 148 and Cys 157 (Supplementary Fig. 7). In the crystal, EL2 plays a central role in lattice contacts, packing against a neighboring Fab with an $870 \AA^{2}$ interface (Supplementary Fig. 6). Because 43 residues were deleted from EL2 in the dDAT length EL2 in transporter structure and function (Supplementary Fig. 8b). Together with EL2, EL4 harbors a $\mathrm{Zn}^{2+}$-binding site in mammalian DATs that modulates transport ${ }^{27}$. The equivalent residues in $\mathrm{dDAT}_{\text {cryst }}$ are within a Ca-Cadistance of $10 \AA$, yet because their identities are Glu 161, Leu 374, and Ala 395, they do not form a high affinity zinc binding site in $\mathrm{dDAT}_{\text {cryst. }}$. 


\section{Tricyclic antidepressant binding site}

Unambiguous density for nortriptyline in $\mathrm{dDAT}_{\text {cryst }}$ was observed in the primary site, approximately halfway across the membrane bilayer (Fig. 2a). In accordance with previous chimeric studies, swapping TM regions between NET and DAT ${ }^{28}$, the drug-binding site is surrounded primarily by helices $1,3,6$ and 8 in a region equivalent to the substrate binding pocket of LeuT ${ }^{15}$, and in close proximity to the densities for sodium and chloride ions (Fig. 2a). The dibenzocycloheptene ring of nortriptyline is oriented as a saddle, curving around the central region of TM3 and engaging in hydrophobic interactions with Val 120, Tyr 124 and Ala 117 (Fig. 2b). Val 120 is extensively conserved and faces the cycloheptene ring (Supplementary Fig. 4a), and replacement of the corresponding Ile 172 in human SERT with larger substitutions such as methionine markedly reduce affinity towards most NSS inhibitors ${ }^{19}$. This location was previously found in human SERT to be protected from crosslinking agents in the presence of inhibitor or substrate ${ }^{29}$. Phe 325 in TM6b forms a $\pi$ stack with one of the benzyl groups of nortriptyline. Residues Gly 425 (TM8) and Ala 479 (TM10) also interact with the tricyclic group of the drug. The $N$-methylpropylamine group of the drug extends across the width of the drug binding site and prevents TMs $1 \mathrm{~b}$ and $6 \mathrm{a}$ from closing the extracellular gate, 'above' the drug. The amine group forms a hydrogen bond with the main chain carbonyl of Phe 43 and a cation- $\pi$ interaction with the side chain of Phe 43 (Fig. 2b). Interestingly, residues equivalent to Val 120 and Phe 43 (Ile 172 and Tyr 95) in SERT are necessary for interactions with antidepressants ${ }^{30}$.

The biogenic amine transporters harbor a crucial aspartate residue in TM1 and in the $\mathrm{dDAT}_{\text {cryst }}$ structure we see how Asp 46 substitutes for the absence of the carboxylate group in biogenic amines as compared to amino acid substrates transported by LeuT, GATs and GlyT (Supplementary Fig. 8a) ${ }^{15}$. The side chain of Asp 46 forms a hydrogen bond with the hydroxyl of Tyr 124, which is equivalent to Tyr 108 in LeuT, which plays a role in substrate recognition $^{31}$ (Fig. 2c). Mutations at this aspartate result in substantial losses in transport activity and reduced binding affinities for cocaine ${ }^{32}$. Ser 421 (TM8), which coordinates a sodium ion at site 2 , is within $3.5 \AA$ to the propylamine group of the TCA and also forms a hydrogen bond with the carbonyl of Phe 43. Ser 421 therefore participates in a network of hydrogen bonds that interconnects nortriptyline with the $\mathrm{Na} 2$ site and was also found to be crucial for high affinity recognition of antidepressants by human SERT ${ }^{33}$.

The $N$-methyl group of nortriptyline is $3.2 \AA$ away from the main chain carbonyl of Phe 319 and sterically prevents Phe 319 and TM6a from closing the extracellular gate above the drug, thereby stabilizing the outward-open state of the transporter. Phe 319 is the equivalent of Phe 253 in LeuT, which gates the substrate binding pocket (Fig. 2c) $)^{15-17}$. The relative position of Phe 319 is markedly different from Phe 253 in the substrate-bound, occluded structure of LeuT, and instead resembles the positions of Phe 253 in the substrate-free and inhibitor-bound structures. To address the question of whether nortriptyline could bind to $\mathrm{dDAT}_{\text {cryst }}$ in a LeuT-like, occluded conformation, we superimposed $\mathrm{dDAT}_{\text {cryst }}$ onto the occluded state of LeuT and found that Phe 319 and Phe 325 would clash with the dibenzocycloheptene ring of the TCA (Fig. 2c, Supplementary Fig. 4c). Identification of nortriptyline bound in the substrate binding pocket of $\mathrm{dDAT}_{\text {cryst }}$ provides the first structural evidence that TCA antidepressants inhibit neurotransmitter transporters by preventing substrate binding and stabilizing the outward open conformation ${ }^{18,19,34}$. The $\mathrm{dDAT}_{\text {cryst }^{-}}$ NTT complex, together with the LeuBAT-antidepressant complexes ${ }^{35}$, conclusively demonstrate that antidepressants inhibit NSSs by acting at the primary or S1 site, in stark contrast to how TCAs inhibit LeuT via a non competitive mechanism ${ }^{36}$ by binding within the extracellular vestibule ${ }^{36-38}$. 


\section{lon-binding sites}

Locations of ions essential for transport could be identified in $\mathrm{dDAT}_{\text {cryst }}$ with electron densities $(>4.0 \sigma)$ at three locations near the non helical hinge-like regions of TMs 1 and 6, and close to the TCA. Densities at the two sites coincided exactly with $\mathrm{Na} 1$ and Na2 sites identified in LeuT (Fig. 3a, b) ${ }^{15}$. A chloride ion was positioned at the third position of high omit density nestled in between TMs 2, 6 and 7 and close to Na1 (Fig. 3a). Placing ions in the omit densities during model building led to a concomitant loss of $F o-F c$ density during refinement. The atomic displacement factors of the ions matched the $B$-values of surrounding atoms. The sodium at site 1 is located $\sim 5.2 \AA$ away from the amino group of nortriptyline and is coordinated with an octahedral geometry by side chain oxygens of Asn 49, Ser 320, Asn 352 and main chain carbonyls of Ala 44 and Ser 320 (Fig. 3a, Supplementary Fig. 8a). Interestingly, the sodium at $\mathrm{Na} 1$ is also coordinated by one water molecule which in turn is within hydrogen bonding distance to Asp 46, thus showing that the Asp in TM1 indirectly participates in the sodium ion coordination. The mean ion coordinating distances $(2.7 \AA)$ at this site are longer than the distances $(2.42 \AA)$ reported for $\mathrm{Na}^{+}$ions in solution but shorter than the distances reported for $\mathrm{K}^{+}$ions $(2.84 \AA$; Supplementary Table 3$)^{39}$.

The chloride ion is located $5.0 \AA$ away from the Na1 site at a position previously identified by computational and mutational studies based on $\operatorname{LeuT}^{40}$ and $\mathrm{GATs}^{41}$. A recent structural study of a chloride-dependent E290S mutant of LeuT also identified a chloride ion at this location ${ }^{42}$. Chloride is coordinated in a tetrahedral fashion through residues in TM6 (Ser 320, Gln 316), TM7 (Ser 356) and TM2 (Tyr 69) (Fig. 3a). Interestingly, the hydroxyl group of Ser 320 bridges the $\mathrm{Na} 1$ and $\mathrm{Cl}^{-}$sites and is positioned to interact with both ions. The mean ion-ligand distances at the $\mathrm{Cl}^{-}$site are $3.0 \AA$ (Supplementary Table 3) and the $B$ factors of surrounding atoms are similar to that of chloride, supporting the placement of chloride at this site.

The sodium at the Na2 site is located 'below' the plane of the drug toward the cytoplasmic face, in between TMs 1 and 8 , and is coordinated in a trigonal bipyramidal fashion by main chain carbonyls from Gly 42 (TM1a), Val 45 (TM1-hinge), Leu 417 (TM8) and the side chain oxygens from Ser 421 and Asp 420 (TM8) (Fig. 3b). The mean ion-oxygen distances are $2.4 \AA$, in line with reported values for sodium coordination in solution (Supplementary Table 3). While the interconnected network of interactions between TMs 1, 6, nortriptyline, sodium, and chloride provides a structure-based mechanism for the coupling of ion and inhibitor binding ${ }^{43}$, we do not have a comprehensive understanding of the ion dependence of inhibitor binding in NSSs.

\section{Cholesterol binding site}

A cholesterol molecule is lodged in a trough-shaped cavity bordered by TM5, TM7, and TM1a at a depth equivalent to the inner leaflet of the membrane (Fig. 4a). Branched aliphatic residues are primarily involved in forming the protein-cholesterol interface (357 $\AA^{2}$ ), thus allowing cholesterol to bury $\sim 57 \%$ of its solvent accessible surface area. Fo-FC density for this site clearly demarcated the orientation of the isooctyl group of cholesterol anchored at the junction of TMs 5 and 7 by residues Leu 276, Leu 277, and Ile 358. The $\beta$ face of the sterol ring primarily faces residues Tyr 273, Leu 270 and Trp 266 in TM5 and also interacts with residues Val 34, Leu 37, Leu 38, and Ile 41 on TM1a. The a-face of cholesterol interfaces with residues Leu 347 and Ile 351 in TM7.

Cholesterol plays an important role in modulating the function of NSS members ${ }^{44,45}$, stabilizing an outward-open state of DAT with a concomitant increase in $B_{\max }$ for cocaine ${ }^{46}$. 
In LeuT, TM1a undergoes a large conformational change upon transition from the outwardfacing open and occluded states to the inward-open state ${ }^{17}$. If a similar conformational change were to occur in dDAT, it would entirely disrupt the cholesterol site (Fig. 4b). We hypothesize that one mechanism for the action of cholesterol on dDAT is that by occupying its binding site in the outward-open, inhibitor-bound state, cholesterol stabilizes the outward-open conformation of the transporter ${ }^{46}$.

\section{Extracellular and cytoplasmic gates}

The ion and ligand binding sites in $\mathrm{dDAT}_{\text {cryst }}$ are accessible to solvent from the extracellular face due to the open gate above the primary binding pocket. The distance between Tyr 124 of TM3 and Phe 319 in TM6a is $10 \AA$, whereas in the substrate-bound occluded state of LeuT the corresponding distance is half as long (Fig. 5a, b). Similarly, the $10 \AA$ separation between Arg 52 on TM1b and Asp 475 on TM10 renders the primary binding site accessible to extracellular solution. The steric bulk of the tricyclic moiety combined with the extended $\mathrm{N}$-methylpropylamine chain of nortriptyline prevents both TM1b and TM6a from approaching TM3 and TM8 to cap the putative substrate pocket and close the gate.

In contrast to the extracellular gate, extensive polar interactions at the intracellular face of the transporter form a thick barrier of $\sim 24 \AA$ between the ligand and ion pockets and solvent to keep the cytoplasmic gate shut. At the cytoplasmic face of the transporter, the indole nitrogen of Trp 30 caps the carbonyl oxygen of Tyr 331 in TM6b, and Arg 27 forms a salt bridge to Asp 435 of TM8 (Fig. 5c). Arg 27, Trp 30, and Asp 435 are strictly conserved in NSS orthologs and LeuT, suggesting that these intracellular gate interactions are general and important facets of the transport mechanism for this family of sodium symporters ${ }^{47,48}$. Tyr 334, the residue corresponding to Tyr 335 in human DAT, was previously shown to be responsible for shifting the conformational equilibrium of the dopamine transporter towards an inward-open state ${ }^{49}$.

\section{C-terminal latch}

Two novel attributes at the C-terminus of $\mathrm{dDAT}_{\text {cryst }}$ were immediately evident from the structure. Helix $12 \mathrm{~b}$ is shifted by $22^{\circ}$ in comparison to its position in LeuT, resulting in the exposure of TM3 to solvent and lipid (Supplementary Fig. 5). Pro 572, conserved in most eukaryotic NSS members, is likely at the root of this kink, and thus plays an important role orienting the second half of the helix away from the rest of the transporter. The hairpin between TM12b and the intracellular C-terminus of $\mathrm{dDAT}_{\text {cryst }}$ is stabilized by hydrogenbonding between the $\varepsilon$-nitrogen of Arg 589 and carbonyl oxygen of Thr 582 (Fig. 5d). The second feature is the C-terminal helix, which contains $2 \frac{1}{2}$ turns from residues 586 to 595 , where several hydrogen bonds and a p-cation interaction between Trp 597 and Arg 101 restrain this C-terminal helix near IL1 at the cytoplasmic face of $\mathrm{dDAT}_{\text {cryst }}$. Although sequence conservation within TM12 and the C-terminus is rather low across NSS orthologs, Gly 584 is strictly conserved and only Lys or Arg is present at the position equivalent to Arg 589 of $\mathrm{dDAT}_{\text {cryst }}$, suggesting that the redirection of the $\mathrm{C}$-terminus in the structure is a conserved feature with functional relevance. Studies of human DAT have identified the region following TM12 to contain sites for protein kinase C-mediated endocytic trafficking ${ }^{3,50}$, and it is plausible that phosphorylation may alter the conformation or accessibility of the C-terminus, allowing it to interact with cellular machinery for internalization. We also note that latch participates in interactions with IL1, which in turn interacts with TM1a, thus suggesting that the C-terminal latch may modulate transporter activity. 


\section{Conclusion}

The structure of $\mathrm{dDAT}_{\text {cryst }}$ captures the transporter in an inhibitor-bound, outward-open conformation. The TCA nortriptyline targets the primary substrate site and stabilizes the open conformation by sterically preventing closure of the extracellular gate (Fig. 6a). One chloride and two sodium ions are located adjacent to the ligand, suggesting that the binding of ions and inhibitor are directly coupled. A cholesterol molecule bound to a crevice flanking TM1a likely stabilizes the outward-open, inhibitor-bound conformation (Fig. 6b). The structure reveals a C-terminal latch that makes extensive interactions with the cytoplasmic face of the transporter, proximal to the cytoplasmic gate, and thus in a position to modulate transport activity. Taken together, the structure of a eukaryotic dopamine transporter reveals novel insights into antidepressant recognition and structural elements implicated in the regulation of neurotransmitter transport, providing a foundation for drug design strategies.

\section{Methods summary}

The $\mathrm{dDAT}_{\text {cryst }}$ construct (Supplementary Fig. 1) was expressed in virus-infected mammalian cells and purified by affinity and size-exclusion chromatography. Fab 9D5 was added prior to crystallization along with nortriptyline $(1 \mathrm{mM})$ at a DAT:Fab molar ratio of 1:1.1 and concentrated down to $3 \mathrm{mg} / \mathrm{ml}$. Crystals of the complex were obtained in the presence of $100 \mathrm{mM}$ glycine $\mathrm{pH} 9$ and 38\% PEG 350 MME. The structure was solved by molecular replacement using a poly-alanine model of LeuT (PDB id. 3F3A) and an ensemble of Fab variable and constant domains. Data processing, model building, and refinement were performed using standard crystallographic software (Supplementary Table 1).

\section{Methods}

\section{Screening, construct optimization and protein expression}

The dDAT was selected as a promising candidate for structural studies after screening multiple orthologues of DATs and NETs by fluorescence-detection size-exclusion chromatography (FSEC) ${ }^{51}$. In addition, FSEC was employed to screen other parameters such as detergent efficacy, thermostability, lipid effects, tertiary epitope-specific monoclonal antibodies and sample homogeneity following purification. The DNA encoding the Drosophila melanogaster DAT was provided by Susan Amara and was modified by removal of the first 20 amino acids (D1-20), by a deletion in EL2 (D164-206) and by point mutations to enhance thermostability (V74A V275A V311A L415A G538L) by PCR-based methods. This modified dDAT sequence, deemed $\mathrm{dDAT}_{\text {cryst }}$, was fused to a C-terminal GFP-His 8 tag with a thrombin cleavage site (LVPRGS) in place of residues 602-607. $\mathrm{dDAT}_{\text {cryst }^{-}}{ }^{-\mathrm{GFP}-\mathrm{His}_{8}}$ was produced by virus-mediated expression in mammalian cells ${ }^{52-54}$.

\section{Antibody production}

Monoclonal antibodies against $\mathrm{dDAT}_{\text {cryst }}$ were raised by Dan Cawley (Vector and Gene Therapy Institute, OHSU) using standard methods. Antibodies were screened by fluorescence-based size-exclusion chromatography and western blot to select clones that recognized natively folded $\mathrm{dDAT}_{\text {cryst }}$ protein. Sequencing of Fab regions was performed on mouse hybridoma cells (Fusion Antibodies Ltd.) and on the intact antibody protein by Edman degradation (Mary Ann Gawinowicz, Columbia University). Antibody was purified from hybridoma supernatant using 4-mercapto-ethyl-pyridine resin. Fab protein was generated by papain cleavage of full-length antibody, followed by $\mathrm{F}_{\mathrm{c}}$ capture on Protein A resin and cation exchange. Fab was stored in $20 \mathrm{mM}$ sodium acetate $\mathrm{pH} 5,250 \mathrm{mM} \mathrm{NaCl}$, and $10 \%$ glycerol. 


\section{Purification of dDAT cryst}

Membranes were solubilized in TBS $(20 \mathrm{mM}$ Tris $\mathrm{pH} 8,150 \mathrm{mM} \mathrm{NaCl})$ containing $\mathrm{n}$ dodecyl- $\beta$-D-maltoside (DDM) at a w/w ratio of $0.1 \mathrm{~g}$ detergent per $1 \mathrm{~g}$ membrane. The detergent-soluble fraction was incubated with cobalt-charged metal ion affinity resin, and $\mathrm{dDAT}_{\text {cryst }}{ }^{-\mathrm{GFP}-\mathrm{His}_{8}}$ was eluted with $100 \mathrm{mM}$ imidazole in $20 \mathrm{mM}$ Tris $\mathrm{pH} 8,300 \mathrm{mM}$ $\mathrm{NaCl}, 5 \%$ glycerol, $14 \mu \mathrm{M}$ lipids (1-palmitoyl-2-oleoyl-sn-glycero-3-phosphocholine (POPC): 1-palmitoyl-2-oleoyl-sn-glycero-3-phosphoethanolamine (POPE): 1-palmitoyl-2oleoyl-sn-glycero-3-phospho-(1'-rac-glycerol) (POPG) at a weight ratio of 3:1:1), $1 \mathrm{mM}$ DDM, and $0.1 \mathrm{mM}$ CHS. After thrombin digestion to remove the GFP-his 8 tag, $\mathrm{dDAT}_{\text {cryst }}$ was isolated by size-exclusion chromatography in $20 \mathrm{mM}$ Tris $\mathrm{pH} 8,100 \mathrm{mM} \mathrm{NaCl}, 5 \%$ glycerol, $14 \mu \mathrm{M}$ POPE, $4 \mathrm{mM}$ decyl-maltoside, and $0.1 \mathrm{mM}$ cholesterol hemisuccinate. The purified $\mathrm{dDAT}_{\text {cryst }}$ protein was mixed with Fab 9D5 at a molar ratio of 1:1.1 and used for crystallization trials at $3 \mathrm{mg} / \mathrm{ml}$ in the presence of $1 \mathrm{mM}$ nortriptyline.

\section{Crystallization}

Crystals grew in $100 \mathrm{mM}$ glycine $\mathrm{pH} 9$ and 38\% PEG 350 MME using a drop ratio of $1 \mu \mathrm{l}$ protein and $0.5 \mu \mathrm{l}$ reservoir solution by hanging drop vapor diffusion. Initial crystals appeared at $4{ }^{\circ} \mathrm{C}$ after 2 days, reaching full size after 7 days. Crystals were flash frozen in liquid $\mathrm{N}_{2}$ directly and used for $\mathrm{X}$-ray diffraction data collection.

\section{Structure determination}

X-ray data were collected at the Advanced Photon Source (Argonne National Laboratory, beamline 24-ID-C). Data were indexed, integrated, and scaled using HKL2000 55 (Supplementary Table 1). The structure was solved using molecular replacement, with ensembles of constant (constant domains of heavy and light chains as one set) and variable domains (variable domains of heavy and light chains as a second set) of Fab coordinates in the PDB along with a poly alanine model of LeuT (PDB id. 3F3A). A multi-model search was done using $P H A S E R^{56}$. Initial phases were improved by iterative steps of manual model building, refinement and maximum likelihood density modification using $\mathrm{COOT}^{57}$, PHENIX Refine ${ }^{58}$ and PHENIX Phase and Build ${ }^{59}$, respectively. Multiple rounds of refinement led to the placement of a majority of main chain and side chain atoms for both

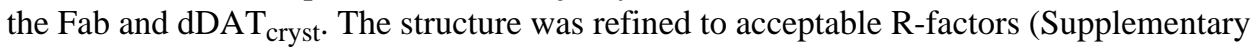
Table 1) with residues 20-24 and 600-605 in $\mathrm{dDAT}_{\text {cryst }}$, and 135-138 in the heavy chain unmodeled due to poor density. Nortriptyline, ions and cholesterol molecules were placed into $F_{o}-F_{c}$ density contoured at $2 \mathrm{~s}$ or greater in the putative substrate pocket, ion sites, and at the periphery of the transporter. Stereochemistry was evaluated using MolProbity ${ }^{60}$.

\section{Ligand binding and uptake measurements}

Scintillation-proximity assays using transporter solubilized in detergent ${ }^{61}$ were carried out using copper yttrium silicate (Cu-YSi) beads (Perkin Elmer) at $0.5 \mathrm{mg} / \mathrm{ml}, 30 \mathrm{nM}^{3} \mathrm{H}-$ labeled nisoxetine $\left(1: 9^{3} \mathrm{H}:{ }^{1} \mathrm{H}\right)$, and $10 \mathrm{nM} \mathrm{dDAT} \mathrm{cryst} \mathrm{GFP}-\mathrm{His}_{8}$ protein in the same buffer as that used for size-exclusion chromatography. Unlabeled nortriptyline was used as the competitor ligand. Assay plates were read using a MicroBeta TriLux 1450 LSC \& Luminescence counter. Data were fitted using a standard single site competition equation, and $K_{i}$ values were calculated from the $\mathrm{IC}_{50}$ values using the Cheng-Prusoff equation.

Uptake assays were performed using human embryonic kidney (HEK 293) cells expressing respective mutant constructs. Cells were resuspended in $10 \mu \mathrm{M}^{3} \mathrm{H}$-dopamine $\left(1: 49^{3} \mathrm{H}:{ }^{1} \mathrm{H}\right)$ containing uptake buffer made with $25 \mathrm{mM}$ Hepes-Tris, pH 7.1, $130 \mathrm{mM} \mathrm{NaCl}, 1 \mathrm{mM}$ $\mathrm{MgSO}_{4}, 5 \mathrm{mM} \mathrm{KCl}, 1 \mathrm{mM} \mathrm{CaCl}_{2}, 5 \mathrm{mM}$ D-glucose and $1 \mathrm{mM} \mathrm{L-ascorbic} \mathrm{acid}{ }^{62}$. Control samples were preincubated with $10 \mu \mathrm{M}$ cold desipramine prior to addition of label. Assays 
were quenched with cold uptake buffer containing $1 \mu \mathrm{M}$ desipramine after $10 \mathrm{~min}$, cells were washed twice with cold uptake buffer and activity was measured from solubilized cells by scintillation counting. Data were plotted using Origin 7.0.

\section{Thermostability screening of $\mathrm{dDAT}_{\text {cryst }}$}

Sites for mutagenesis were selected based on model of dDAT built on the template of LeuT and residues were altered to Ala, Leu, or Phe ${ }^{21}$. Individual mutants along with the wild-type construct were transfected into HEK cells and kept in culture for 48 hours, then tested for binding activity after detergent solubilization. Samples were split and one part was kept at $4^{\circ} \mathrm{C}$, and the other portion of lysate heated at $40^{\circ} \mathrm{C}$ for 10 minutes. ${ }^{3} \mathrm{H}$-nisoxetine was added prior to heating to select for mutants that stabilize an inhibitor-bound state of the transporter. Scintillation proximity assay was used to monitor activity in a high-throughput format. Mutants that consistently had an increased melting temperature $(\mathrm{Tm})$ compared to wild-type $\left(\operatorname{Tm}=35^{\circ} \mathrm{C}\right)$ were chosen and pooled into one construct, which yielded a 5 mutant construct with a $T m$ of $\sim 60^{\circ} \mathrm{C}$.

\section{Supplementary Material}

Refer to Web version on PubMed Central for supplementary material.

\section{Acknowledgments}

We thank Dr. Dan Cawley (VGTI, OHSU) for generating monoclonal antibodies and Susan Amara for providing the wild-type dDAT construct. We would like to thank H. Wang and D. Claxton for comments and suggestions along with other Gouaux lab members for their helpful discussion during manuscript preparation. We thank L. Vaskalis for assistance with figures and H. Owen for help with manuscript preparation. We thank the staff of the Northeastern Collaborative Access Team (NECAT) at the Advanced Photon Source (APS) for assistance with data collection. This work was supported by a postdoctoral fellowship from the American Heart Association (A.P.), a National Institute of Mental Health research award (K.H.W.) and by the NIH (E.G.) E.G. is an investigator with the Howard Hughes Medical Institute.

\section{References}

1. Jessell TM, Kandel ER. Synaptic transmission: a bidirectional and self-modifiable form of cell-cell communication. Cell. 1993; 72(Suppl):1-30. [PubMed: 8381334]

2. Masson J, Sagne C, Hamon M, El Mestikawy S. Neurotransmitter transporters in the central nervous system. Pharmacol Rev. 1999; 51:439-464. [PubMed: 10471414]

3. Kristensen AS, et al. SLC6 neurotransmitter transporters: structure, function, and regulation. Pharmacol Rev. 2011; 63:585-640. [PubMed: 21752877]

4. Rudnick G. Ion-coupled neurotransmitter transport: thermodynamic vs. kinetic determinations of stoichiometry. Methods Enzymol. 1998; 296:233-247. [PubMed: 9779452]

5. Radian R, Bendahan A, Kanner BI. Purification and identification of the functional sodium- and chloride-coupled gamma-aminobutyric acid transport glycoprotein from rat brain. J Biol Chem. 1986; 261:15437-15441. [PubMed: 3536902]

6. Waldman ID, et al. Association and linkage of the dopamine transporter gene and attention-deficit hyperactivity disorder in children: heterogeneity owing to diagnostic subtype and severity. Am J Hum Genet. 1998; 63:1767-1776. [PubMed: 9837830]

7. Shannon JR, et al. Orthostatic intolerance and tachycardia associated with norepinephrinetransporter deficiency. N Engl J Med. 2000; 342:541-549. [PubMed: 10684912]

8. Meldrum BS. Neurotransmission in epilepsy. Epilepsia. 1995; 36(Suppl 1):S30-S35. [PubMed: 23057108]

9. Kurian MA, et al. Homozygous loss-of-function mutations in the gene encoding the dopamine transporter are associated with infantile parkinsonism-dystonia. J Clin Invest. 2009; 119:15951603. [PubMed: 19478460] 
10. Kuhn R. The treatment of depressive states with G 22355 (imipramine hydrochloride). Am J Psychiatry. 1958; 115:459-464. [PubMed: 13583250]

11. Axelrod J, Whitby LG, Hertting G. Effect of psychotropic drugs on the uptake of H3norepinephrine by tissues. Science. 1961; 133:383-384. [PubMed: 13685337]

12. Berton O, Nestler EJ. New approaches to antidepressant drug discovery: beyond monoamines. Nat Rev Neurosci. 2006; 7:137-151. [PubMed: 16429123]

13. Pletscher A. The discovery of antidepressants: a winding path. Experientia. 1991; 47:4-8. [PubMed: 1999242]

14. Anderson IM. Selective serotonin reuptake inhibitors versus tricyclic antidepressants: a metaanalysis of efficacy and tolerability. J Affect Disord. 2000; 58:19-36. [PubMed: 10760555]

15. Yamashita A, Singh SK, Kawate T, Jin Y, Gouaux E. Crystal structure of a bacterial homologue of

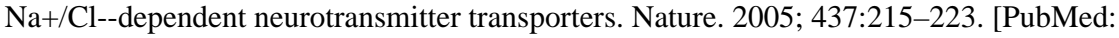
16041361]

16. Singh SK, Piscitelli CL, Yamashita A, Gouaux E. A competitive inhibitor traps LeuT in an opento-out conformation. Science. 2008; 322:1655-1661. [PubMed: 19074341]

17. Krishnamurthy H, Gouaux E. X-ray structures of LeuT in substrate-free outward-open and apo inward-open states. Nature. 2012; 481:469-474. [PubMed: 22230955]

18. Beuming $\mathrm{T}$, et al. The binding sites for cocaine and dopamine in the dopamine transporter overlap. Nat Neurosci. 2008; 11:780-789. [PubMed: 18568020]

19. Sorensen L, et al. Interaction of antidepressants with the serotonin and norepinephrine transporters: mutational studies of the S1 substrate binding pocket. J Biol Chem. 2012; 287:43694-43707. [PubMed: 23086945]

20. Porzgen P, Park SK, Hirsh J, Sonders MS, Amara SG. The antidepressant-sensitive dopamine transporter in Drosophila melanogaster: a primordial carrier for catecholamines. Mol Pharmacol. 2001; 59:83-95. [PubMed: 11125028]

21. Serrano-Vega MJ, Magnani F, Shibata Y, Tate CG. Conformational thermostabilization of the beta1-adrenergic receptor in a detergent-resistant form. Proc Natl Acad Sci U S A. 2008; 105:877882. [PubMed: 18192400]

22. Tatsumi M, Groshan K, Blakely RD, Richelson E. Pharmacological profile of antidepressants and related compounds at human monoamine transporters. Eur J Pharmacol. 1997; 340:249-258. [PubMed: 9537821]

23. Torres GE, et al. Oligomerization and trafficking of the human dopamine transporter Mutational analysis identifies critical domains important for the functional expression of the transporter. $\mathrm{J}$ Biol Chem. 2003; 278:2731-2739. [PubMed: 12429746]

24. Sitte HH, Farhan H, Javitch JA. Sodium-dependent neurotransmitter transporters: oligomerization as a determinant of transporter function and trafficking. Mol Interv. 2004; 4:38-47. [PubMed: 14993475]

25. Li LB, et al. The role of $\mathrm{N}$-glycosylation in function and surface trafficking of the human dopamine transporter. J Biol Chem. 2004; 279:21012-21020. [PubMed: 15024013]

26. Chen R, et al. Direct evidence that two cysteines in the dopamine transporter form a disulfide bond. Mol Cell Biochem. 2007; 298:41-48. [PubMed: 17131045]

27. Norregaard L, Frederiksen D, Nielsen EO, Gether U. Delineation of an endogenous zinc-binding site in the human dopamine transporter. EMBO J. 1998; 17:4266-4273. [PubMed: 9687495]

28. Buck KJ, Amara SG. Structural domains of catecholamine transporter chimeras involved in selective inhibition by antidepressants and psychomotor stimulants. Mol Pharmacol. 1995; 48:1030-1037. [PubMed: 8848002]

29. Chen JG, Sachpatzidis A, Rudnick G. The third transmembrane domain of the serotonin transporter contains residues associated with substrate and cocaine binding. J Biol Chem. 1997; 272:2832128327. [PubMed: 9353288]

30. Henry LK, et al. Tyr-95 and Ile-172 in transmembrane segments 1 and 3 of human serotonin transporters interact to establish high affinity recognition of antidepressants. J Biol Chem. 2006; 281:2012-2023. [PubMed: 16272152] 
31. Bismuth Y, Kavanaugh MP, Kanner BI. Tyrosine 140 of the gamma-aminobutyric acid transporter GAT-1 plays a critical role in neurotransmitter recognition. J Biol Chem. 1997; 272:16096-16102. [PubMed: 9195904]

32. Kitayama S, et al. Dopamine transporter site-directed mutations differentially alter substrate transport and cocaine binding. Proc Natl Acad Sci U S A. 1992; 89:7782-7785. [PubMed: 1502198]

33. Andersen $\mathrm{J}$, et al. Location of the antidepressant binding site in the serotonin transporter: importance of Ser-438 in recognition of citalopram and tricyclic antidepressants. J Biol Chem. 2009; 284:10276-10284. [PubMed: 19213730]

34. Talvenheimo J, Fishkes H, Nelson PJ, Rudnick G. The serotonin transporter-imipramine "receptor". J Biol Chem. 1983; 258:6115-6119. [PubMed: 6853478]

35. Wang H, Gouaux E. Structural basis for conserved mechanism of action by chemically diverse antidepressants on neurotransmitter sodium symporters. manuscript in preparation. 2013

36. Singh SK, Yamashita A, Gouaux E. Antidepressant binding site in a bacterial homologue of neurotransmitter transporters. Nature. 2007; 448:952-956. [PubMed: 17687333]

37. Zhou Z, et al. LeuT-desipramine structure reveals how antidepressants block neurotransmitter reuptake. Science. 2007; 317:1390-1393. [PubMed: 17690258]

38. Zhou Z, et al. Antidepressant specificity of serotonin transporter suggested by three LeuT-SSRI structures. Nat Struct Mol Biol. 2009; 16:652-657. [PubMed: 19430461]

39. Harding MM. Metal-ligand geometry relevant to proteins and in proteins: sodium and potassium. Acta Crystallogr D Biol Crystallogr. 2002; 58:872-874. [PubMed: 11976508]

40. Forrest LR, Tavoulari S, Zhang YW, Rudnick G, Honig B. Identification of a chloride ion binding site in $\mathrm{Na}+/ \mathrm{Cl}$-dependent transporters. Proc Natl Acad Sci U S A. 2007; 104:12761-12766. [PubMed: 17652169]

41. Zomot E, et al. Mechanism of chloride interaction with neurotransmitter:sodium symporters. Nature. 2007; 449:726-730. [PubMed: 17704762]

42. Kantcheva AK, et al. Chloride binding site of neurotransmitter sodium symporters. Proc Natl Acad Sci U S A. 2013; 110:8489-8494. [PubMed: 23641004]

43. Tavoulari S, Forrest LR, Rudnick G. Fluoxetine (Prozac) binding to serotonin transporter is modulated by chloride and conformational changes. J Neurosci. 2009; 29:9635-9643. [PubMed: 19641126]

44. Scanlon SM, Williams DC, Schloss P. Membrane cholesterol modulates serotonin transporter activity. Biochemistry. 2001; 40:10507-10513. [PubMed: 11523992]

45. North P, Fleischer S. Alteration of synaptic membrane cholesterol/phospholipid ratio using a lipid transfer protein Effect on gamma-aminobutyric acid uptake. J Biol Chem. 1983; 258:1242-1253. [PubMed: 6822499]

46. Hong WC, Amara SG. Membrane cholesterol modulates the outward facing conformation of the dopamine transporter and alters cocaine binding. J Biol Chem. 2010; 285:32616-32626. [PubMed: 20688912]

47. Bennett ER, Su H, Kanner BI. Mutation of arginine 44 of GAT-1, a $(\mathrm{Na}(+)+\mathrm{Cl}(-))$-coupled gamma-aminobutyric acid transporter from rat brain, impairs net flux but not exchange. J Biol Chem. 2000; 275:34106-34113. [PubMed: 10926932]

48. Cao Y, Li M, Mager S, Lester HA. Amino acid residues that control pH modulation of transportassociated current in mammalian serotonin transporters. J Neurosci. 1998; 18:7739-7749. [PubMed: 9742144]

49. Loland CJ, Norregaard L, Litman T, Gether U. Generation of an activating Zn(2+) switch in the dopamine transporter: mutation of an intracellular tyrosine constitutively alters the conformational equilibrium of the transport cycle. Proc Natl Acad Sci U S A. 2002; 99:1683-1688. [PubMed: 11818545]

50. Holton KL, Loder MK, Melikian HE. Nonclassical, distinct endocytic signals dictate constitutive and PKC-regulated neurotransmitter transporter internalization. Nat Neurosci. 2005; 8:881-888. [PubMed: 15924135]

51. Kawate T, Gouaux E. Fluorescence-detection size-exclusion chromatography for precrystallization screening of integral membrane proteins. Structure. 2006; 14:673-681. [PubMed: 16615909] 
52. Dukkipati A, Park HH, Waghray D, Fischer S, Garcia KC. BacMam system for high-level expression of recombinant soluble and membrane glycoproteins for structural studies. Protein Expr Purif. 2008; 62:160-170. [PubMed: 18782620]

53. Reeves PJ, Callewaert N, Contreras R, Khorana HG. Structure and function in rhodopsin: highlevel expression of rhodopsin with restricted and homogeneous $\mathrm{N}$-glycosylation by a tetracyclineinducible N-acetylglucosaminyltransferase I-negative HEK293S stable mammalian cell line. Proc Natl Acad Sci U S A. 2002; 99:13419-13424. [PubMed: 12370423]

54. Baconguis I, Gouaux E. Structural plasticity and dynamic selectivity of acid-sensing ion channelspider toxin complexes. Nature. 2012; 489:400-405. [PubMed: 22842900]

55. Otwinowski Z, Minor W. Processing of X-ray diffraction data collected in oscillation mode. Meth. Enzymol. 1997; 276:307-326.

56. McCoy AJ, et al. Phaser crystallographic software. J Appl Crystallogr. 2007; 40:658-674. [PubMed: 19461840]

57. Emsley P, Cowtan K. Coot: model-building tools for molecular graphics. Acta Crystallogr D Biol Crystallogr. 2004; 60:2126-2132. [PubMed: 15572765]

58. Afonine PV, et al. Towards automated crystallographic structure refinement with phenix.refine. Acta Crystallogr D Biol Crystallogr. 2012; 68:352-367. [PubMed: 22505256]

59. Terwilliger TC, et al. Iterative model building, structure refinement and density modification with the PHENIX AutoBuild wizard. Acta Crystallogr D Biol Crystallogr. 2008; 64:61-69. [PubMed: 18094468]

60. Chen VB, et al. MolProbity: all-atom structure validation for macromolecular crystallography. Acta Crystallogr D Biol Crystallogr. 2010; 66:12-21. [PubMed: 20057044]

61. Quick M, Javitch JA. Monitoring the function of membrane transport proteins in detergentsolubilized form. Proc Natl Acad Sci U S A. 2007; 104:3603-3608. [PubMed: 17360689]

62. Giros B, et al. Cloning, pharmacological characterization, and chromosome assignment of the human dopamine transporter. Mol Pharmacol. 1992; 42:383-390. [PubMed: 1406597] 
a

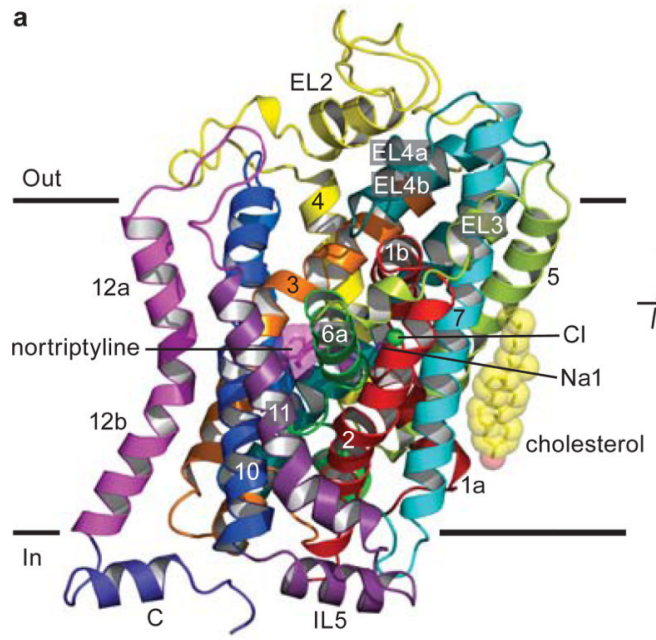

b

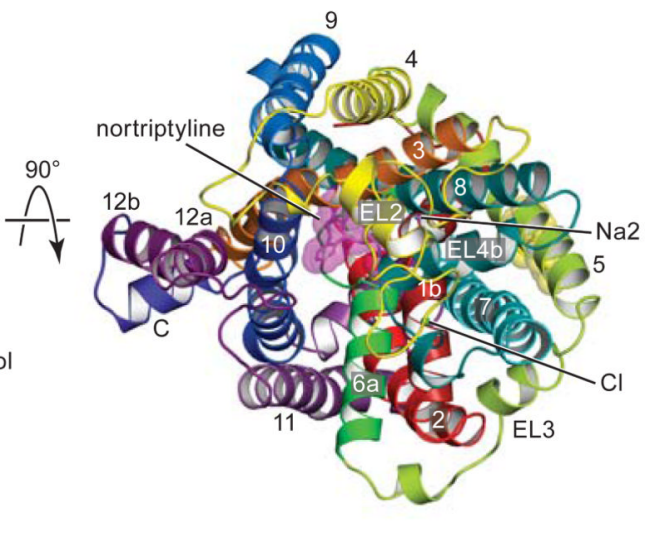

c

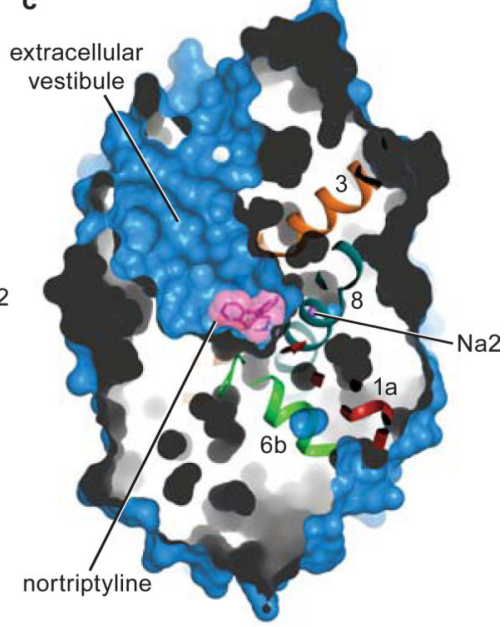

Figure 1. Architecture of dDAT cryst $_{\text {t }}$

a, Structure of $\mathrm{dDAT}_{\text {cryst }}$ viewed parallel to membrane. Nortriptyline, sodium ions, a chloride ion, and a cholesterol molecule are shown in sphere representation in magenta,

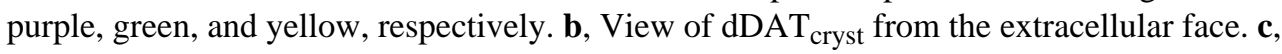
Surface representation showing that ligand and ion binding sites are accessible from the extracellular vestibule. Nortriptyline and TMs 1, 3, 6, and 8 are colored as in panel a. 
a

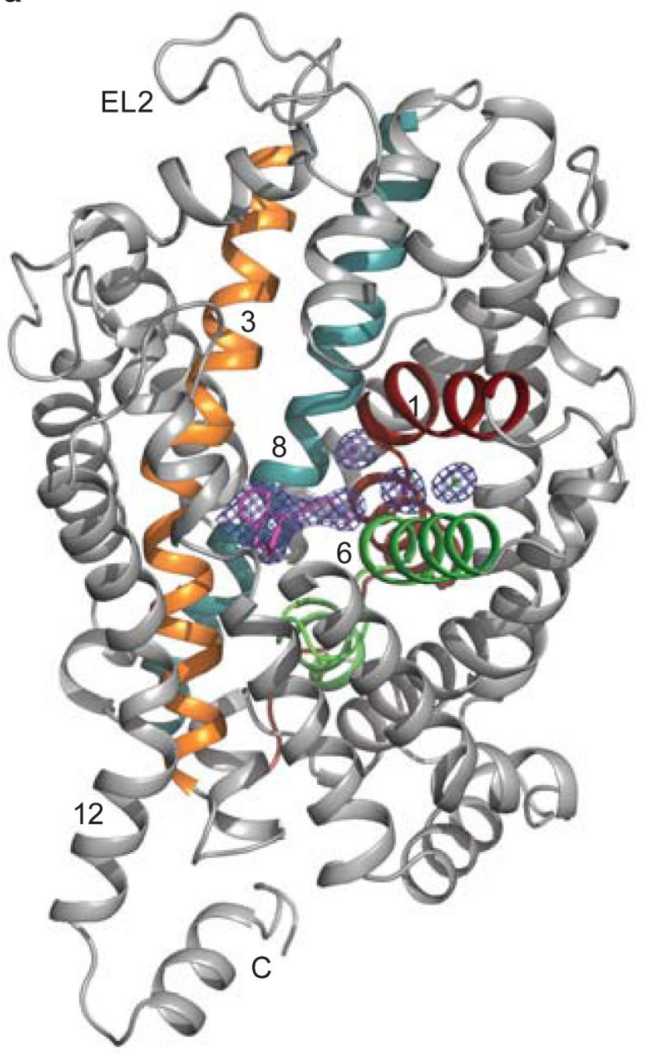

b

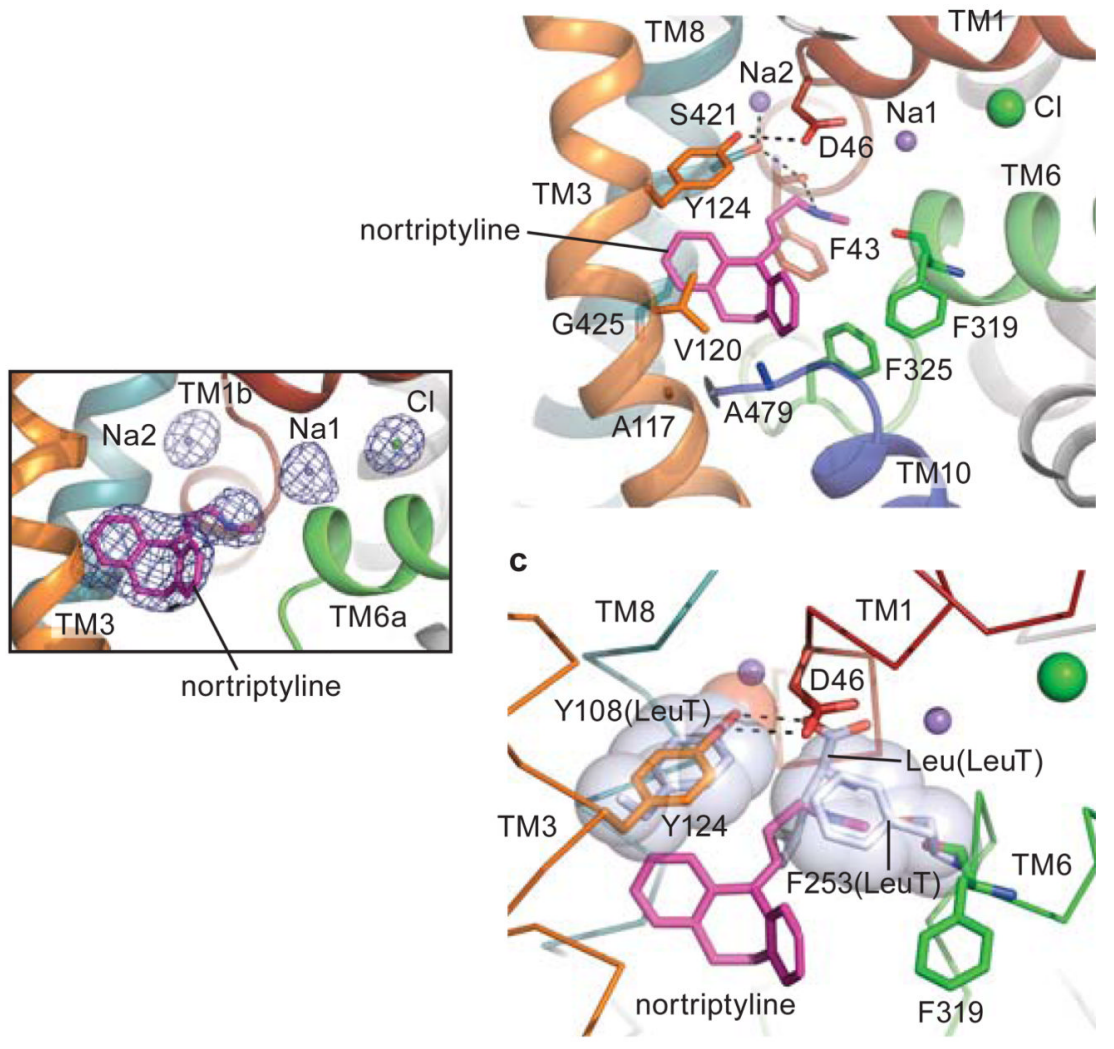

Figure 2. Antidepressant binding site

a, Overall view of the nortriptyline-bound $\mathrm{dDAT}_{\text {cryst }} F o-F c$ densities (blue mesh) for drug and ions are contoured at $\sigma$ levels of 2.5 and 3.0, respectively. b. Close-up view of the drug binding pocket. $\mathrm{Na}^{+}$and $\mathrm{Cl}$ ions are shown as spheres. Nortriptyline is represented as sticks (magenta). The amino group of nortriptyline is $2.8 \AA$ from the carbonyl oxygen of F43 (TM1a) and the $N$-methyl group of nortriptyline is $3.2 \AA$ from the carbonyl oxygen of F319. Residues lining the drug binding pocket with interfacial areas greater than $10 \AA^{2}$ are represented as sticks. c. Comparison of the drug or substrate-binding pocket of $\mathrm{dDAT}_{\text {cryst }}$ with that of LeuT (PDB id. 2A65), respectively. The distance between the carboxylate group of leucine and Tyr 108 (spheres) is $2.7 \AA$ in the occluded state (2A65) and $5.1 \AA$ in the inhibitor-bound state $(3 \mathrm{~F} 3 \mathrm{~A})$ of LeuT, whereas the equivalent interaction in $\mathrm{dDAT}_{\text {cryst }}$ between Asp 46 and Tyr 124 is $3.2 \AA$. 
a

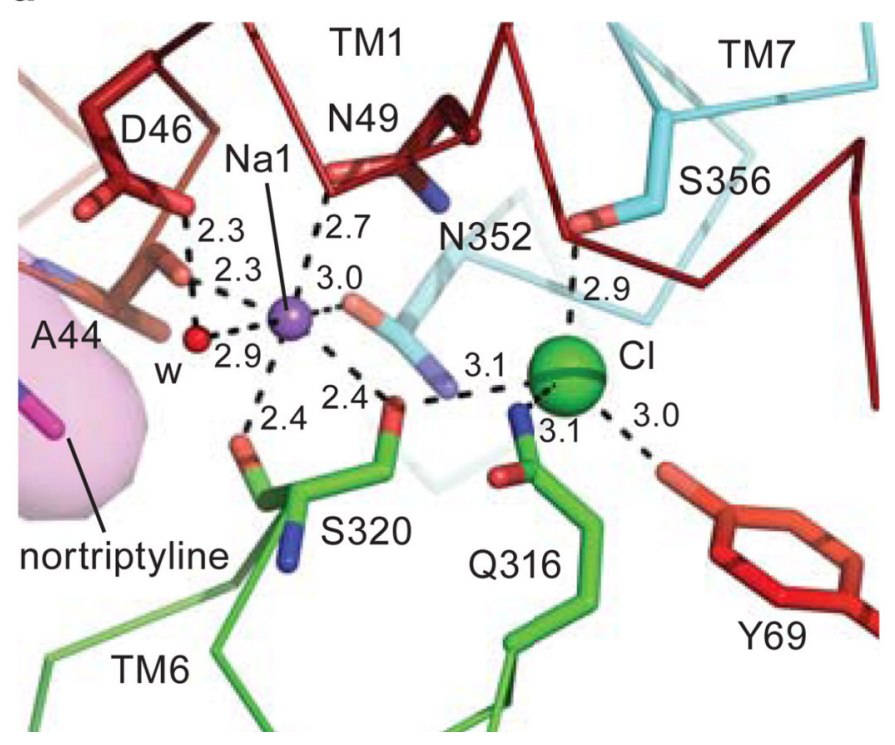

b

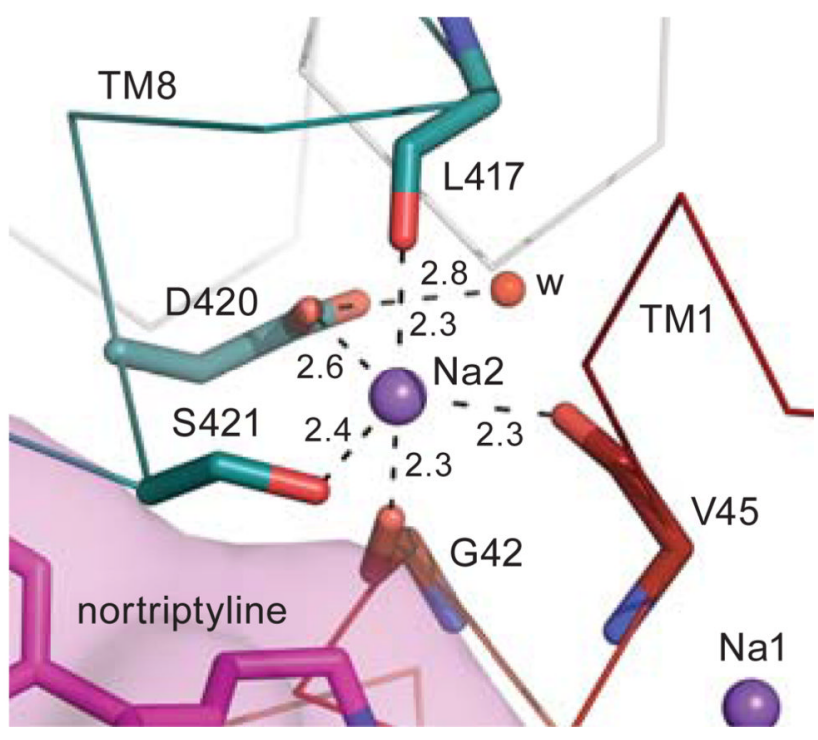

Figure 3. Ion binding sites

a, $\mathrm{Na} 1$ and chloride ion-binding sites. $\mathrm{Na}^{+}$is purple and $\mathrm{Cl}$ is green and both are modeled as spheres. b. Coordination at the $\mathrm{Na} 2$ site. Coordination at the site is trigonal bipyramidal with the water molecule (red sphere) $3.3 \AA$ from the sodium ion. Distances are in ängstroms for residues that are in the coordination sphere and interactions are shown by dashed lines.

Residues are colored according to their respective TMs. 
a

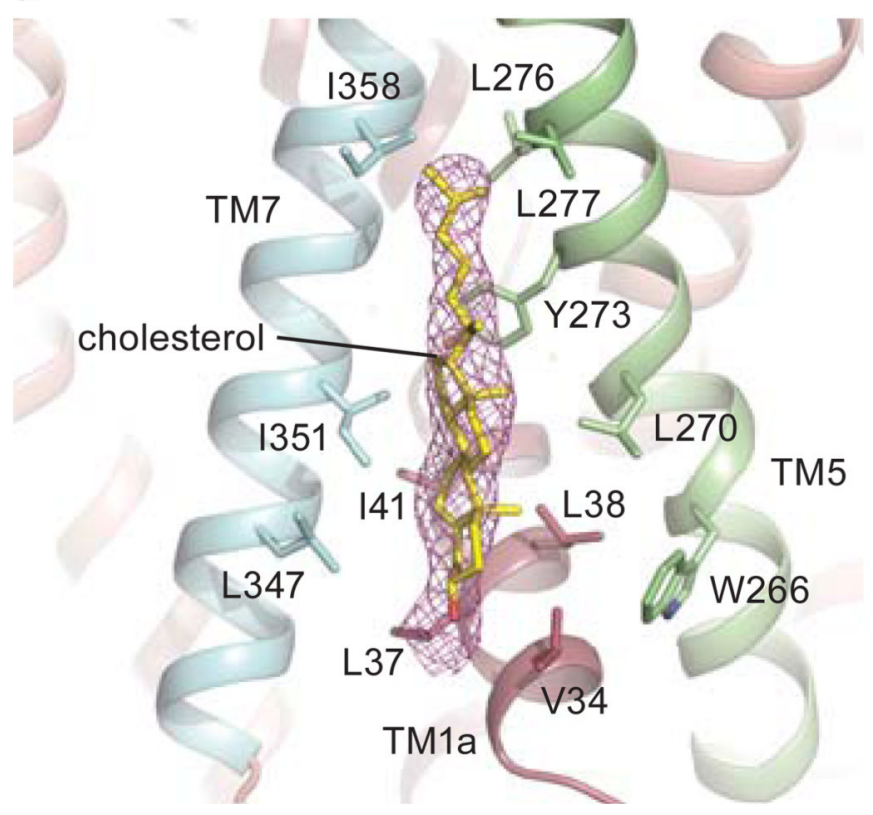

b

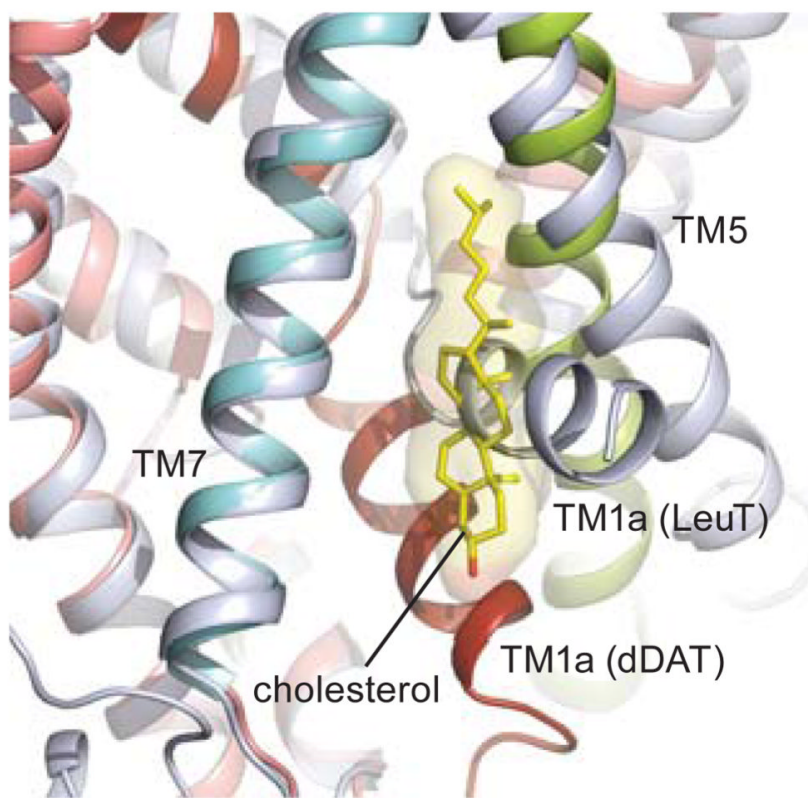

Figure 4. Cholesterol site

a, Cholesterol (yellow sticks) shown with $F o-F c$ density (light magenta) contoured at $2.0 \sigma$. Residues that interface with the cholesterol group are represented as sticks. b. Potential role of cholesterol in maintaining an outward-open state of transporter. Cholesterol (sticks with transparent surface) sterically clashes with the position of TM1a in the inward-open conformation of LeuT (PDB id. 3TT3) ${ }^{17}$. 
a

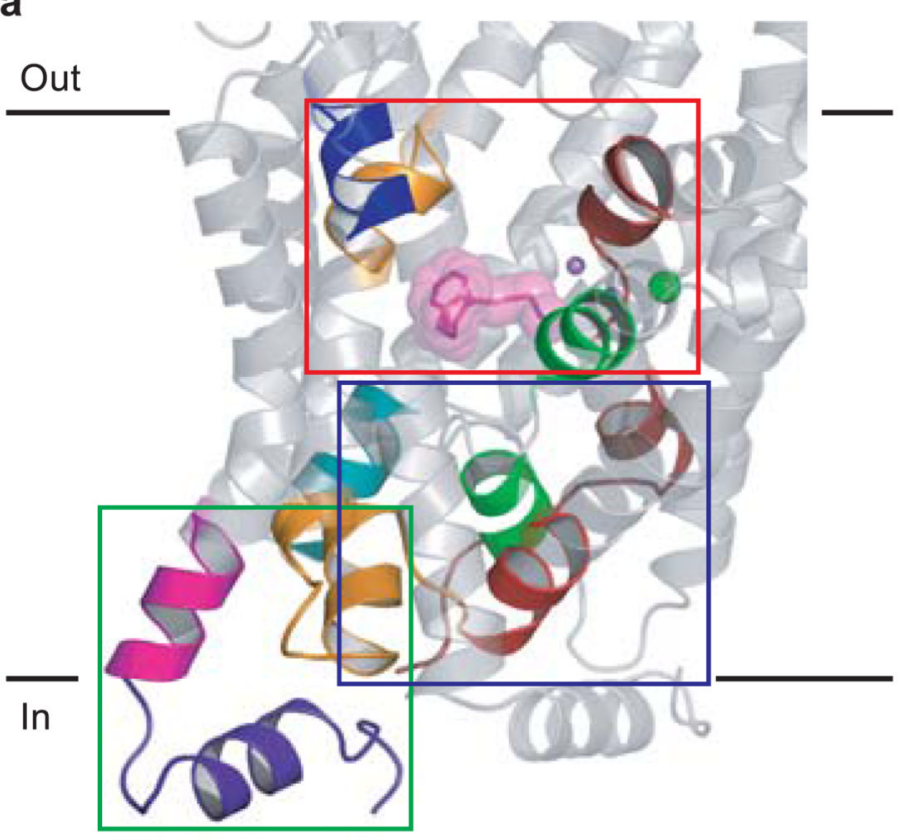

b

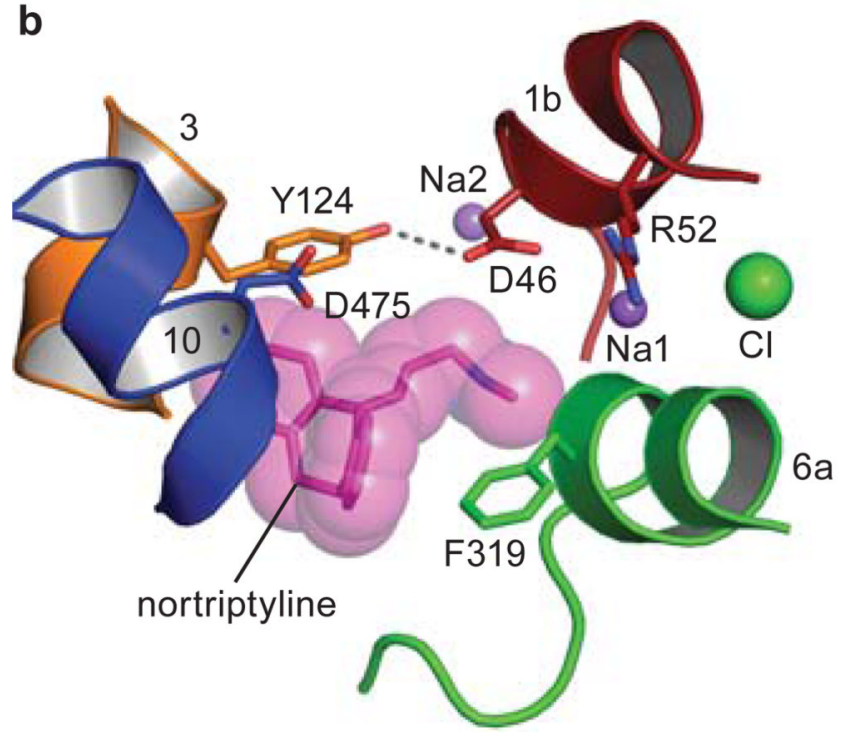

C

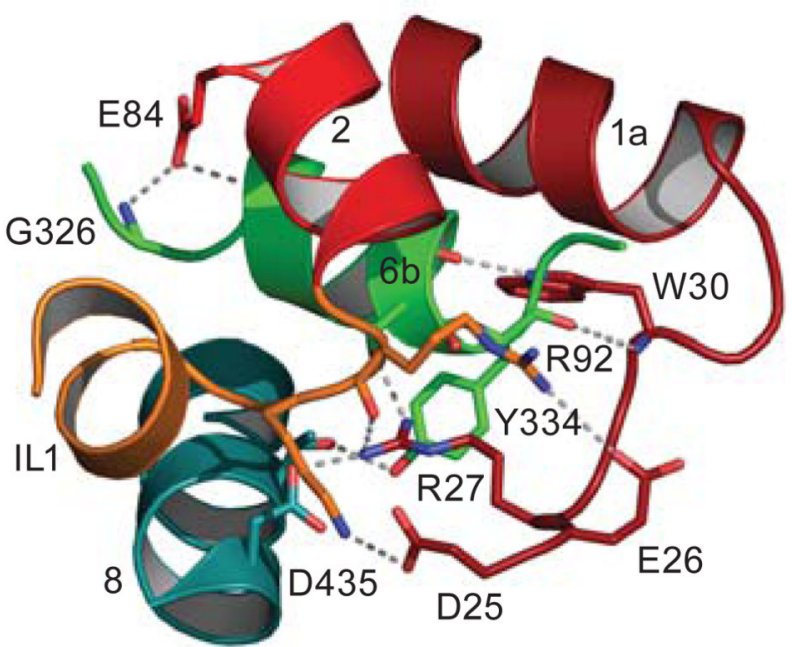

d

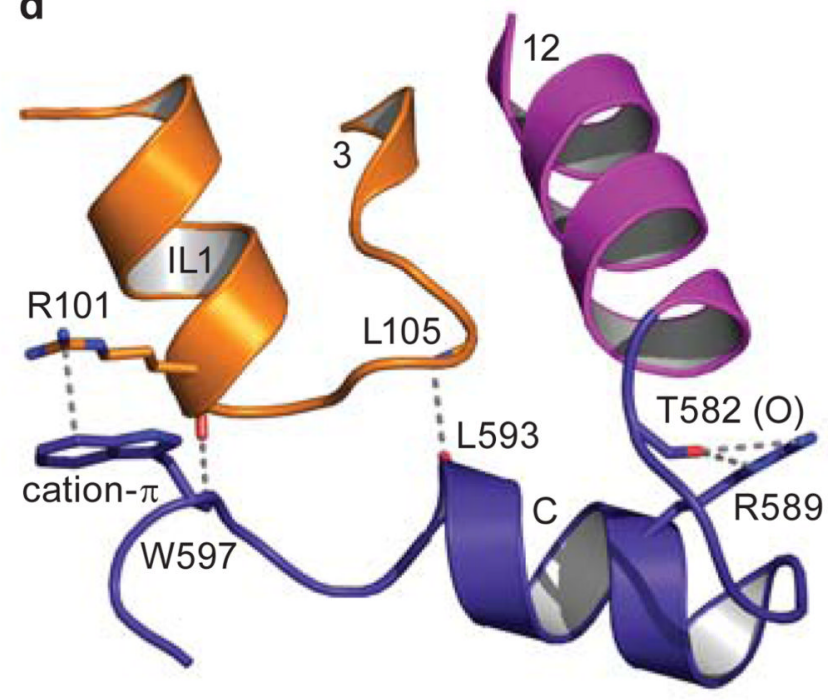

Figure 5. Extracellular and cytoplasmic gates and the C-terminal latch

a, Relative locations of the open extracellular gate (red box), closed cytoplasmic gate (blue box), and C-terminal latch (green box) in $\mathrm{dDAT}_{\text {cryst }} \cdot \mathbf{b}$, The width of the extracellular gate is depicted by the distances between Y124 and F319 (10 ̊), and R52 and D475 (10 ̊).

Nortriptyline, ions, and helices are colored as in figure 1. c, The cytoplasmic gate is closed by polar and electrostatic interactions between TM1a, TM2, IL1, TM6b, and TM8. d, The C-terminal helix following TM12 is bound to the cytoplasmic face of the transporter via polar interactions with IL1. Polar and electrostatic bonds are represented as gray dashed lines. 
a

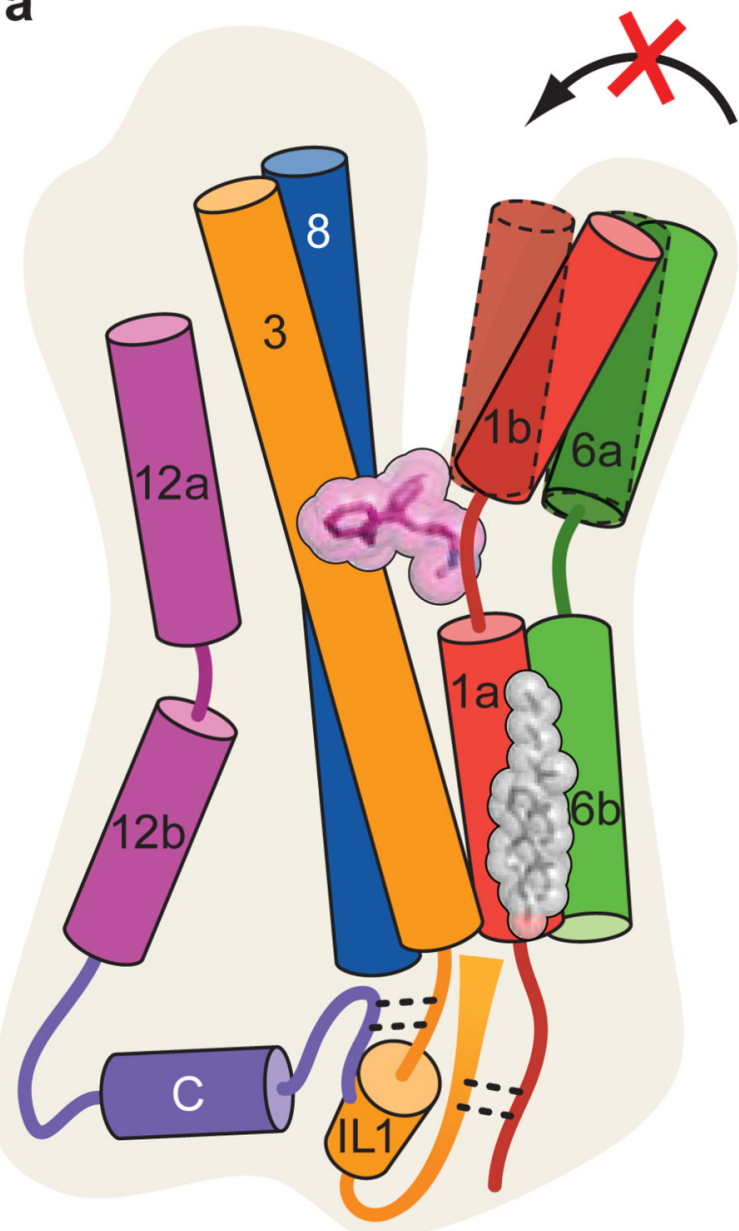

b

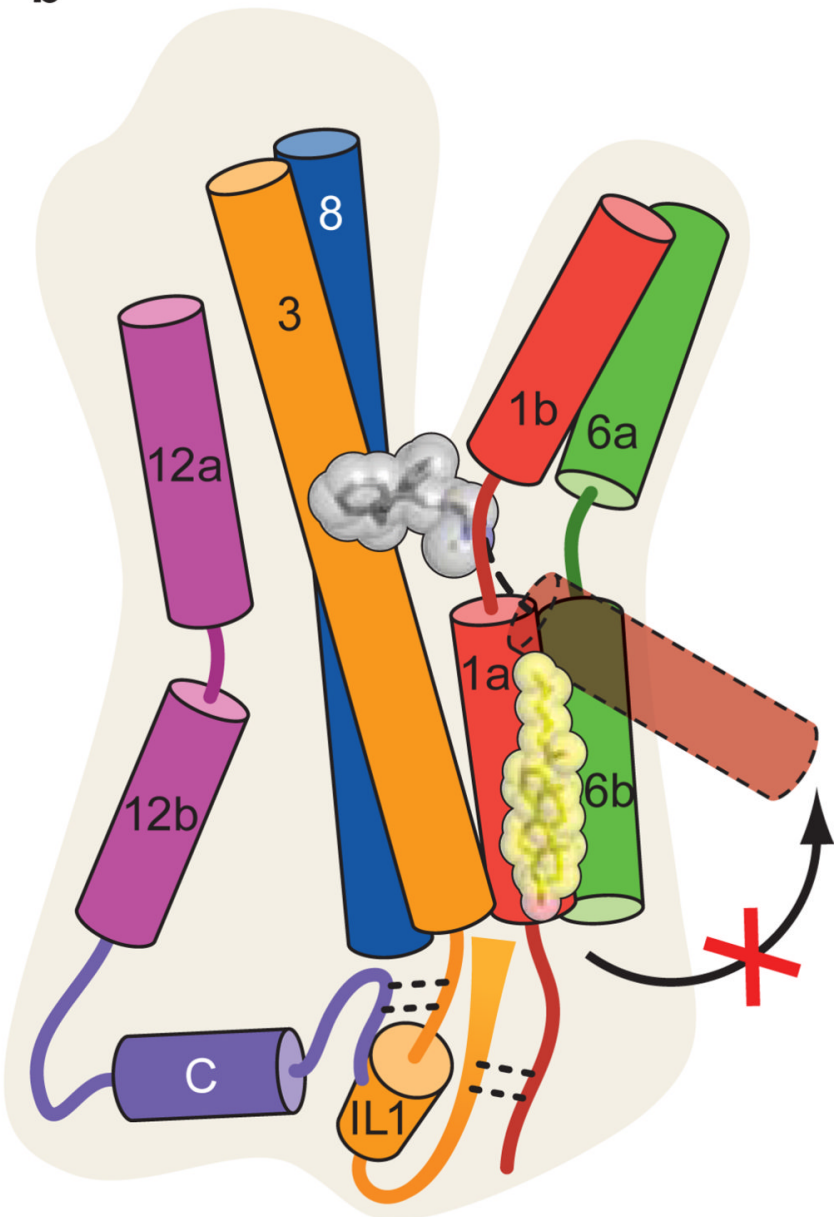

Figure 6. Mechanisms of antidepressants and cholesterol

a, The TCA nortriptyline (magenta) wedges between scaffold helices 3 and 8 and the core helices 1 and 6 , preventing the movement of TMs $1 \mathrm{~b}$ and $6 \mathrm{a}$ from closing the extracellular vestibule. b. Cholesterol (yellow) is bound in an intracellular pocket and prevents the movement of TM1a from opening, thereby stabilizing the outward-open conformation of DAT. The C-terminal latch interacts with IL1 as part of the cytoplasmic gate. 